\title{
Investigation a single-spiral vortex in a swirl flow
}

\author{
Elizaveta S. Gesheva ${ }^{1,}$, Sergey I. Shtork ${ }^{1,2}$, and Sergey V. Alekseenko ${ }^{1,2}$ \\ ${ }^{1}$ Kutateladze Institute of Thermal Physics, Siberian Branch of Russian Academy of Sciences, \\ Lavrentyev Ave. 1, Novosibirsk, 630090 Russia \\ ${ }^{2}$ Novosibirsk State University, Pirogova Str. 2, Novosibirsk, 630090 Russia
}

\begin{abstract}
The work is aimed at a detailed study of large-scale helical vortex structures emerging in a high turbulent intensively swirling flow. It was shown that the vortex formed in the chamber by installing diaphragm with shifted outlet is folded into a single-helical vortex. The flow visualization shows that although the vortex axis performs slow oscillations (precession), on average this structure is fixed in space. The velocity fields were measured with the aid of a nonintrusive method of flow diagnostics (PIV). Verification of the calculation results obtained using a LES simulation was performed based on measured experimental data to confirm the correctness of the chosen mathematical modelling approach. It has been asserted that the investigated regimes are in a selfsimilarity area relative to Reynolds number.
\end{abstract}

\section{Introduction}

The application of swirling flows and useful properties of vortex structures has been grounded scientifically and widely used in practice [1]. Longitudinal concentrated vortices increase the efficiency of devices and hardware systems. Stationary helical vortex structures were first observed at the Institute of Thermophysics of the SB RAS [2]. Until now, there is no quantitative information on such vortices that hinders understanding the physics of formation of vortices and developing approaches for mathematical description of the vortex dynamics.

Setting up the experiments with complex swirling flow is a hard task and often it is not feasible to extract enough data, so the numerical modeling plays an important role. The Navier-Stokes equations describing the motion of a viscous fluid are among the most important instruments in hydrodynamics and are used in the mathematical modeling of many natural phenomena and technical processes [3]. Currently, the numerical simulation methods are rapidly developing. Different approaches in the modeling of turbulence make it possible to expand the notion of fluid motion including swirling flows with the formation of complex vortex structures. To verify the simulation results, a joint experimental and numerical study of the vortex structures is used, analogous to $[4,5]$

\footnotetext{
* Corresponding author: gesheva es@itp.nsc.ru
} 


\section{Experimental method}

To carry out the experiments, a hydrodynamic stand created earlier in the IT SB RAS [2] to study turbulent swirling flow has been modernized. The hydrodynamic stand (Fig. 1) is used basically for experimental study of the vortex structures formed in a working chamber 1 with the tangential flow inlet. Water circulating through a closed hydraulic circuit was used as a working fluid. The liquid from the tank 2 is fed into the working chamber by means of a pump 3, through a system of valves and flow distributors. The liquid flow rate is measured by an ultrasonic flowmeter (5 - electronic module, 6 - measuring section of the flowmeter).
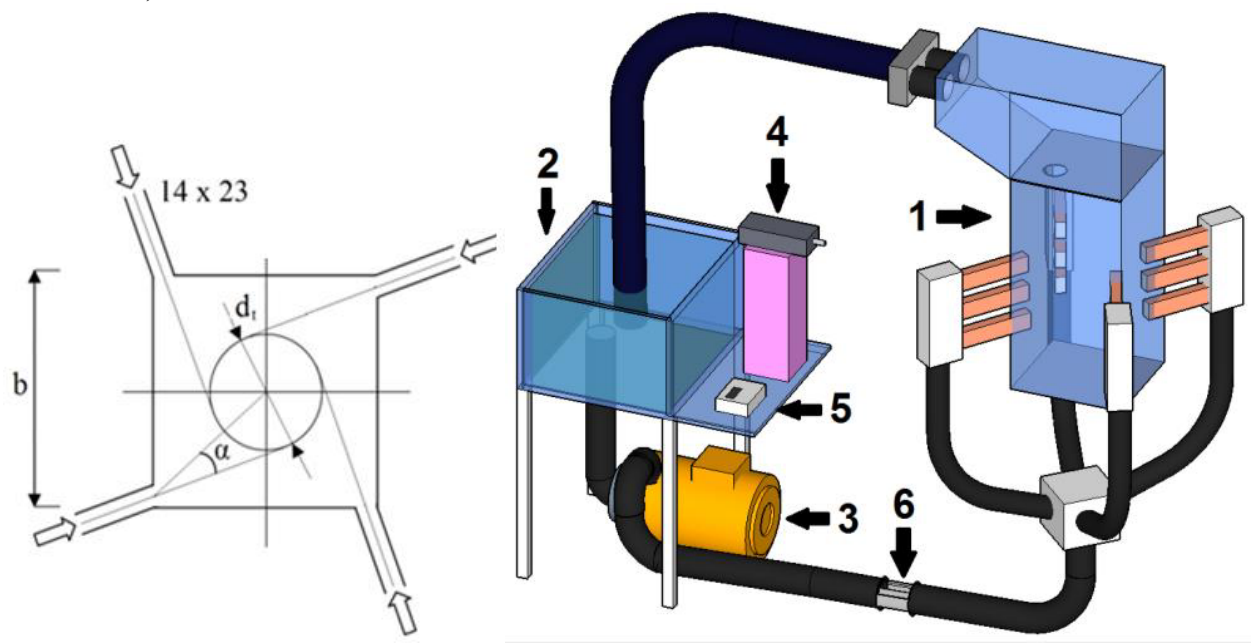

Fig. 1. Scheme of the vortex chamber.

The test section is made of plexiglas as a vertical channel of square cross-section with dimensions $188 \times 188 \times 560 \mathrm{~mm}$. Rectangular nozzles with an output sizes of $14 \times 23 \mathrm{~mm}$ are arranged in three tiers and are combined into four corner blocks. The swirling flow was organized by tangential fluid supply to the work area, i.e. by directing the axes of the nozzles along the tangent to some conditional circle with a diameter $d_{\mathrm{t}}($ Fig. 1).

The flow regimes were characterized by dimensionless parameters, such as the Reynolds number Re and the swirl parameter $S$. The Reynolds number was determined using the transverse dimension of the channel $b$ and the average velocity $V_{b}$ in the vortex chamber:

$$
\mathrm{Re}=\mathrm{bV}_{\mathrm{b}} / \mathrm{v}
$$

The swirl parameter was defined through the swirler geometry and varied by changing the angle of rotation of the nozzles $\alpha$ (the diameter of the conditional circle $d_{t}$ ) [4]:

$$
\mathrm{S}=\mathrm{bd}_{\mathrm{t}} / \Sigma_{\mathrm{n}}
$$

where $\Sigma_{\mathrm{n}}$ - area of the inlet nozzles. The experiments included flow visualization, which was organized using a light source and by small air bubbles that were fed into the circuit in front of the working area. The flow pattern was recorded using a high-resolution digital mirror camera with the video recording function at a frequency of 60 frames per second. To obtain quantitative characteristics of the vortex, such as velocity fields, a nonintrusive PIV system (Particle Image Velocimetry) was applied. 


\section{Characteristics of a spiral vortex}

Visualization studies have shown that when the geometry of the chamber changes, various vortex structures are formed. In the chamber with a flat bottom and a centrally located diaphragm orifice, a rectilinear vortex is formed from the bottom of the chamber to the outlet. When the diaphragm is displaced, the vortex filament is bended into a spiral (Figure 2).

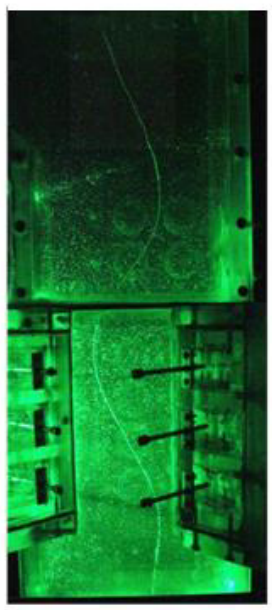

$\operatorname{Re}=20000$

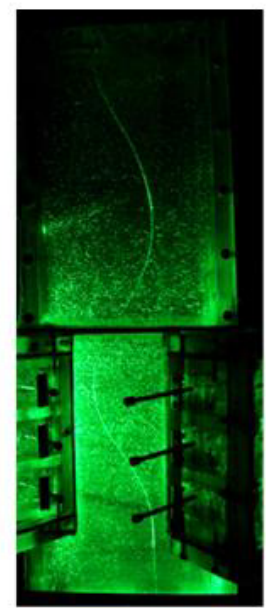

$\operatorname{Re}=23000$

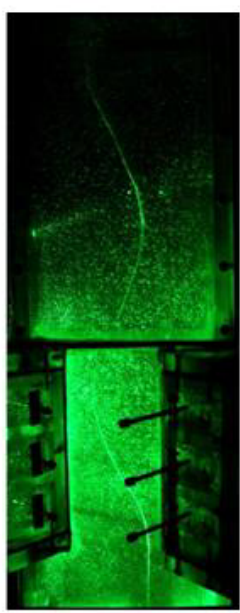

$\operatorname{Re}=27000$

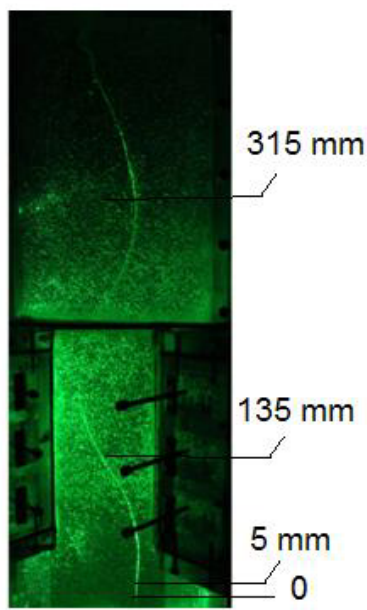

$\operatorname{Re}=37000$

Fig. 2. Visualization of spiral vortex at different Reynolds numbers, $S=8,4$.

Figure 2 demonstrates that form of the spiral vortex is unchanged for all studied Reynolds numbers. Although analysis of the video recording shows that the vortex axis executes slow oscillations relative to the average position, but in general this structure is stationary. The fluid particles move around the spiral axis, thus making a double spiral motion. Also can be noticed some areas with a reverse flow.

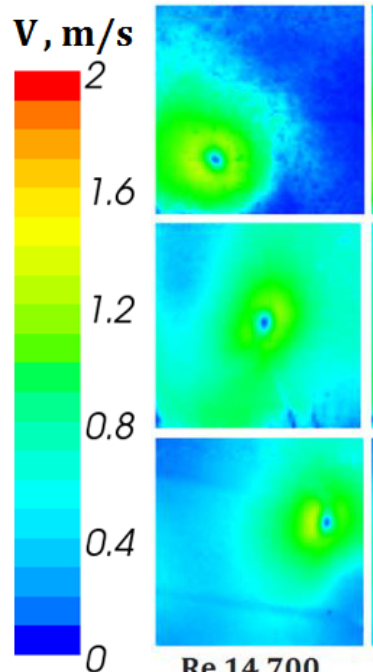

Re 14700
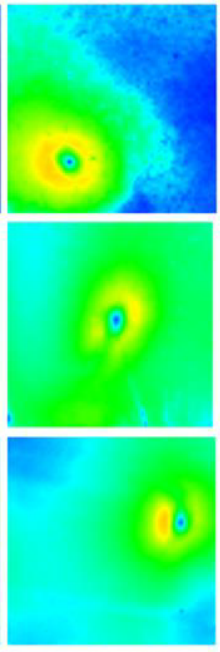

Re 19200
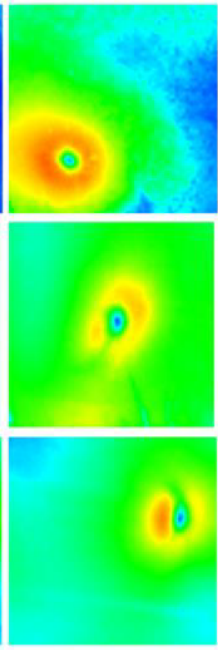

Re 23600
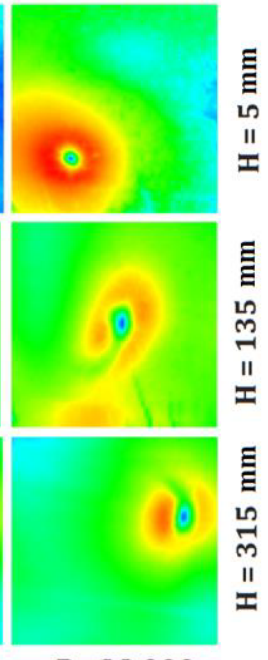

Re 28000

Fig. 3. Velocity fields in the cross sections of the chamber, $S=8,4$. 
Figure 3 shows the velocity fields in and around the vortex core at different horizontal cross-sections of the chamber. The plots demonstrate formation of a localized vortex core at all studied Reynolds numbers.
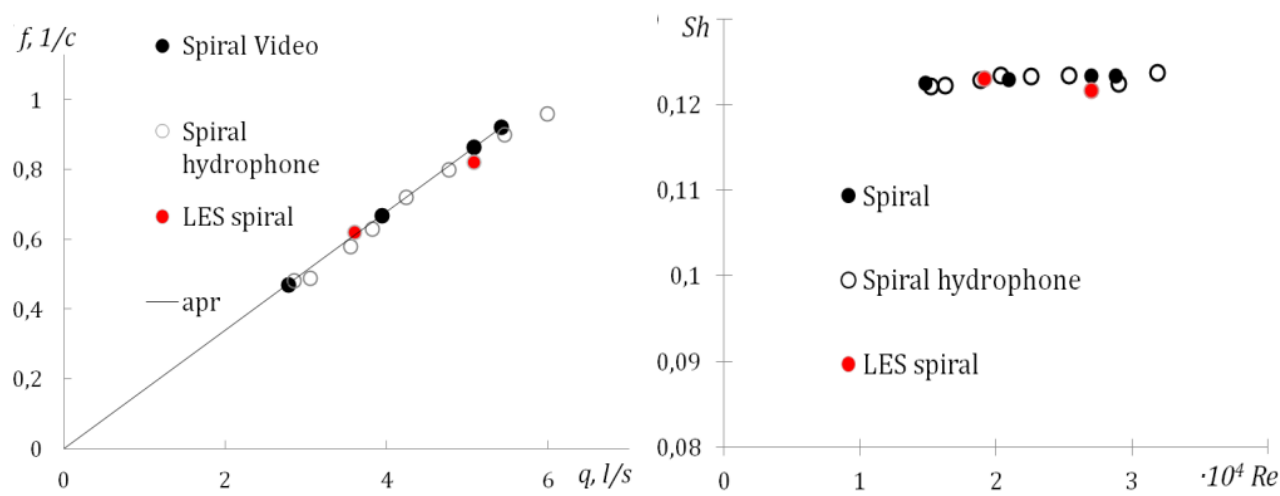

Fig. 4. Frequency dependence of the precession of a vortex on flow rate (left). The dependence of Strouhal number on Reynolds number (right), $S=8,4$.

Detailed analysis of the video recordings in a horizontal plane allowed to reveal that the mentioned above oscillations of the vortex core axis have a form of precession. Figure 4 shows the dependence of the precession frequency on the fluid flow. The frequency of the vortex precession was measured in several ways: based on the analysis of video recordings; using a hydrophone installed on the wall of the channel and based on the numerical simulation using a CFD package by the LES method [6].

\section{Conclusions}

As a result of the investigations it was shown that the geometry of the chamber and the boundary conditions have a key influence on the shape of the vortex axis. E.g. displacement of the outlet leads to the formation of a spiral vortex, which is quasi-stationary although makes small regular oscillations in form of precession. Helical vortex is stable and does not change form with Reynolds number. Strouhal number of the precession also does not depend on the Reynolds number that points on the flow self-similarity. The velocity fields measured using PIV technique confirm that spiral vortex has a localized vortex core.

The study was financially supported by the Russian Science Foundation (project № 14-29-00093).

\section{References}

1. S.V. Alekseenko, V.M. Dulin, M.P. Tokarev, D.M. Markovich, Springer Proceedings in Physics, 185, 43 (2016)

2. S.V. Alekseenko, P.A. Kuibin, V.L. Okulov, S.I. Shtork, J. Fluid Mech. 48, 123 (1999).

3. S. Alimohammadi, E. Fanning, T. Persoons, D.B. Murray, Computers \& Fluids 140, 232 (2016)

4. C.E. Cala, E.C. Fernandes, M.V. Heitor, S.I. Shtork, Exp. Fluids. 40, 267 (2006)

5. D.J. Ryan, M.J.H. Simmons, M.R. Baker, Chem. Eng. Sci. 163, 123 (2017)

6. E.S. Gesheva, S.I. Shtork, S.V. Alekseenko, Vestnik of NSU: Physics. 9, 39 (2014). [in Russian] 